\title{
Shifted Jacobi polynomials for nonlinear singular variable-order time fractional Emden-Fowler equation generated by derivative with non-singular kernel
}

\author{
M.H. Heydari' , Z. Avazzadeh ${ }^{2 *}$ (D) and A. Atangana ${ }^{3,4}$
}

"Correspondence:

zakieh.avazzadeh@xjtlu.edu.cn

${ }^{2}$ Department of Applied

Mathematics, Xi'an

Jiaotong-Liverpool University,

Suzhou 215123, Jiangsu, China

Full list of author information is

available at the end of the article

\begin{abstract}
In this work, a nonlinear singular variable-order fractional Emden-Fowler equation involved with derivative with non-singular kernel (in the Atangana-Baleanu-Caputo type) is introduced and a computational method is proposed for its numerical solution. The desired method is established upon the shifted Jacobi polynomials and their operational matrix of variable-order fractional differentiation (which is extracted in the present study) together with the spectral collocation method. The presented method transforms obtaining the solution of the main problem into obtaining the solution of an algebraic system of equations. Several numerical examples are examined to show the validity and the high accuracy of the established method.
\end{abstract}

Keywords: Shifted Jacobi polynomials (SJPs); Operational matrices; Variable-order (VO) time fractional derivative; Singular VO time fractional Emden-Fowler equation

\section{Introduction}

In recent years, fractional calculus (theory of differentiation and integration of arbitrary order) has become an interesting topic for researchers in physics, mathematics and engineering. This is because of the fact that fractional differential equations govern the behavior of several physical systems with more accuracy [1]. It should be noted that main advantage of using fractional differential (integral) equations for modeling applied problems is their non-locality. This means that in a fractional dynamical system, the next state depends on all the previous states. The interested reader is referred to [2-7] for recent advance in the fractional derivatives and their applications.

Recently, the issue of singularity of kernel in fractional operators has motivated the interest of many researchers to introduce other new classes of operators with non-singular kernel, for instance see [8-11]. It is shown that fractional equations involving operators with non-singular kernel capable to model various phenomena in physics and engineering more accurate than the ones with singular kernel [12]. On the other hand, during the last decades, considerable efforts have been devoted to study the variable-order (VO) fractional calculus (derivative and integral operators of variable orders) and its applications in

(c) The Author(s) 2021. This article is licensed under a Creative Commons Attribution 4.0 International License, which permits use, sharing, adaptation, distribution and reproduction in any medium or format, as long as you give appropriate credit to the original author(s) and the source, provide a link to the Creative Commons licence, and indicate if changes were made. The images or other third party material in this article are included in the article's Creative Commons licence, unless indicated otherwise in a credit line to the material. If material is not included in the article's Creative Commons licence and your intended use is not permitted by statutory regulation or exceeds the permitted use, you will need to obtain permission directly from the copyright holder. To view a copy of this licence, visit http://creativecommons.org/licenses/by/4.0/. 
science and engineering; see e.g. [13-15]. Due to these facts, there has been great attention on the approximate methods for the numerical solution of problems involving $\mathrm{VO}$ fractional derivatives with non-singular kernel. In [16], the authors proposed a meshless technique for a class of $2 \mathrm{D}$ reaction-diffusion equations involving $\mathrm{VO}$ fractional differentiations with non-singular kernel. The authors of [17] utilized the artificial neural networks for some categories of differential equations involved with VO fractional derivatives with non-singular kernel. A numerical method established in [18] for a category of VO reaction-diffusion equations involving differentiations with non-singular kernel. The authors of [19] established a finite difference scheme for a category of telegraph equations involving $\mathrm{VO}$ fractional operators with non-singular kernel. An interpolation method using the Lagrange polynomials applied in [20] for solving some classes of differential equations with this kind of $\mathrm{VO}$ fractional derivatives. The moving least square method is used in [21] for a category of $\mathrm{VO}$ fractional 2D telegraph equations including derivatives with non-singular kernel. A wavelet method utilized in [22] for a category of VO fractional 2D reaction-diffusion equations including derivatives with non-singular kernel. In [23], the author applied a cardinal method for a class of nonlinear VO fractional optimal control problems generated by this kind of derivatives. Legendre cardinal functions are used in [24] for solving VO fractional Schrödinger equation defined by Atangana-Baleanu nonsingular derivative.

The Jacobi polynomials, as an important class of orthogonal polynomials have been effectively used in the approximation theory and practical applications [25]. The best-known classes of the Jacobi polynomials are the Legendre polynomials, the first kind, the second kind, the third kind and the fourth kind Chebyshev polynomials [26]. It is worth noting that these polynomials play an important role in establishing the spectral methods [26]. The spectral accuracy of such methods allow one to obtain highly accurate solutions for the problem under consideration. Meanwhile, obtaining operational matrices of fractional and classical differentiation and integration for these polynomials is often easy in comparison with other basis functions. So, in recent years, the Jacobi polynomials have been widely used to solve various classes of problems. In [25], the authors used the Jacobi polynomials for a class of multi-variable-order differential equations. These polynomials have been used in [27] to establish a computational method for generalized fractional Burgers equations. An operational matrix scheme using the Jacobi polynomials was developed in [28] for linear and nonlinear fractional differential equations. These polynomials are utilized in [29] for solving distributed order fractional reaction-diffusion equations. In [30], the fractional forms of these polynomials are used for solving fractional variational problems.

One of the well-known singular differential equations which often arises in physics and engineering is the Emden-Fowler equation. This equation models several physical phenomena, such as the isothermal gas sphere, the thermal behavior of spherical cloud of gas, the theory of stellar structure and the theory of thermionic currents [31-33]. In the last decades, much attention has been focused on the numerical study of this equation and similar generalized equations; for instance, see [34-36] and the references therein.

The main aims of this study are to introduce a generalized version of the Emden-Fowler equation defined by the Atangana-Baleanu-Caputo (A-B-C) VO fractional derivative and to express a computational method for its numerical solution. So, we focus on the 
following equation:

$$
\begin{aligned}
& \frac{\partial^{\omega(\xi, \tau)} u(\xi, \tau)}{\partial \tau^{\omega(\xi, \tau)}}=\frac{\partial^{2} u(\xi, \tau)}{\partial \xi^{2}}+\frac{\lambda}{\xi} \frac{\partial u(\xi, \tau)}{\partial \xi}+\mathcal{G}(\xi, \tau, u(\xi, \tau))+f(\xi, \tau), \\
& \omega(\xi, \tau) \in(0,1),(\xi, \tau) \in[0, L] \times[0, T]
\end{aligned}
$$

where the VO fractional differentiation is defined in the sens of $A-B-C$ sense with the Mittag-Leffler function as its kernel [8], subject to the initial condition

$$
u(\xi, 0)=g(\xi)
$$

and boundary conditions

$$
u(0, \tau)=h_{0}(\tau), \quad u(L, \tau)=h_{1}(\tau)
$$

Here, $\omega(\xi, \tau)$ is a given continuous function, $u(\xi, \tau)$ is the temperature of the system under consideration, $\lambda$ is a constant, $\mathcal{G}(\xi, \tau, u(\xi, \tau))+f(\xi, \tau)$ is the nonlinear source term which assumed to be continuous on its defined domain, and $g(\xi), h_{0}(\tau)$ and $h_{1}(\tau)$ are given continuous functions describing the initial and boundary conditions (IBC).

The proposed algorithm is based upon the shifted Jacobi polynomials (SJPs) and their operational matrices of the classical and VO fractional derivatives which are obtained in this study. More precisely, the desired solution of the considered model is approximated by the SJPs with unknown coefficients. This approximation and its classical and VO fractional derivatives are substituted into the problem. By utilizing the operational matrices of the SJPs a residual function is defined for the problem. By taking some collocation points into the residual function and considering the IBCs, solving the approximated problem is turned into solving an algebraic system of equations.

This paper is arranged as follows: A brief review of non-singular VO fractional calculus is expressed in the next section. The SJPs and some of their properties are introduced in Sect. 3. Some new operational matrices are obtained for the SJPs in Sect. 4. The algorithm is explained in Sect. 5. Several test problems are solved by the proposed approach in Sect. 6. The last section is dedicated to a short conclusion.

\section{Vo fractional calculus with non-singular kernel}

Definition 2.1 ([1]) The Mittag-Leffler function is defined by

$$
\mathbf{E}_{\mu_{1}, \mu_{2}}(\tau)=\sum_{j=0}^{\infty} \frac{\tau^{j}}{\Gamma\left(j \mu_{1}+\mu_{2}\right)}, \quad \tau \in \mathbb{C}, \mu_{1}, \mu_{2} \in \mathbb{R}^{+} .
$$

Note that in the case of $\mu_{2}=1$, it is abbreviated as $\mathbf{E}_{\mu_{1}}(\tau)$.

Definition 2.2 ([8]) Let $\bar{\omega} \in(0,1)$ be a real constant and $u(\xi, \tau)$ be a differentiable function on $[0, L] \times[0, T]$. The fractional derivative of order $\bar{\omega}$ of $u(\xi, \tau)$ with respected to $\tau$ in the $\mathrm{A}-\mathrm{B}-\mathrm{C}$ sense is given by

$$
\frac{\partial^{\bar{\omega}} u(\xi, \tau)}{\partial \tau^{\bar{\omega}}}=\frac{\overline{\mathbf{C}}(\bar{\omega})}{1-\bar{\omega}} \int_{0}^{\tau} \frac{\partial u(\xi, s)}{\partial s} \mathbf{E}_{\bar{\omega}}\left(\frac{-\bar{\omega}(\tau-s)^{\bar{\omega}}}{1-\bar{\omega}}\right) d s,
$$

where $\overline{\mathbf{C}}(0)=\overline{\mathbf{C}}(1)=1$. According to [37] we can define $\overline{\mathbf{C}}(\bar{\omega})=1-\bar{\omega}+\frac{\bar{\omega}}{\Gamma(\bar{\omega})}$. 
Definition $2.3([22,37])$ Suppose that $\omega:[0, L] \times[0, T] \longrightarrow(0,1)$ is continuous and $u(\xi, \tau)$ be differentiable with respect to $\tau$ on $[0, L] \times[0, T]$. The fractional differentiation of order $\omega(\xi, \tau)$ of $u(\xi, \tau)$ in the $\mathrm{A}-\mathrm{B}-\mathrm{C}$ type is given by

$$
\frac{\partial^{\omega(\xi, \tau)} u(\xi, \tau)}{\partial \tau^{\omega(\xi, \tau)}}=\frac{\mathbf{C}(\omega(\xi, \tau))}{1-\omega(\xi, \tau)} \int_{0}^{\tau} \frac{\partial u(\xi, s)}{\partial s} \mathbf{E}_{\omega(\xi, \tau)}\left(\frac{-\omega(\xi, \tau)(\tau-s)^{\omega(\xi, \tau)}}{1-\omega(\xi, \tau)}\right) d s,
$$

where $\mathbf{C}(\omega(\xi, \tau))=1-\omega(\xi, \tau)+\frac{\omega(\xi, \tau)}{\Gamma(\omega(\xi, \tau))}$.

Corollary 2.4 ([22]) If $\omega:[0, L] \times[0, T] \longrightarrow(0,1)$ is continuous and $k \in \mathbb{N} \cup\{0\}$, then

$$
\frac{\partial^{\omega(\xi, \tau)} \tau^{k}}{\partial \tau^{\omega(\xi, \tau)}}= \begin{cases}0, & k=0, \\ \frac{\mathbf{C}(\omega(\xi, \tau)) k ! \tau^{k}}{1-\omega(\xi, \tau)} \mathbf{E}_{\omega(\xi, \tau), k+1}\left(\frac{-\omega(\xi, \tau) \tau^{\omega(\xi, \tau)}}{1-\omega(\xi, \tau)}\right), & k=1,2, \ldots\end{cases}
$$

\section{The shifted Jacobi polynomials (SJPs) and their properties}

The SJPs can be defined over $[0, T]$ by the following explicit analytic formula [38]:

$$
\begin{aligned}
& J_{T, i}^{(a, b)}(\tau)=\sum_{k=0}^{i}(-1)^{i+k} \frac{\Gamma(i+b+1) \Gamma(i+k+a+b+1)}{\Gamma(k+b+1) \Gamma(i+a+b+1)(i-k) ! k ! T^{k}} \tau^{k}, \\
& \quad i \in \mathbb{N} \cup\{0\}, a, b>-1 \text { and } a+b \neq-1 .
\end{aligned}
$$

These polynomials generate a system which is orthogonal with the associated weight function $w_{T}^{(a, b)}(\tau)=\tau^{b}(T-\tau)^{a}$ over $[0, T]$ with the orthogonality property

$$
\int_{0}^{T} J_{T, i}^{(a, b)}(\tau) J_{T, j}^{(a, b)}(\tau) w_{T}^{(a, b)}(\tau) d \tau=h_{T, i}^{(a, b)} \delta_{i j}
$$

where

$$
h_{T, i}^{(a, b)}=\frac{T^{a+b+1} \Gamma(i+a+1) \Gamma(i+b+1)}{(2 i+a+b+1) i ! \Gamma(i+a+b+1)} .
$$

Any function $u(\tau)$, square integrable with respect to $w_{T}^{(a, b)}(\tau)$ over $[0, T]$ can be written by the SJPs as follows:

$$
u(\tau)=\sum_{i=0}^{\infty} c_{i} J_{T, i}^{(a, b)}(\tau)
$$

where

$$
c_{i}=\frac{1}{h_{T, i}^{(a, b)}} \int_{0}^{T} w_{T}^{(a, b)}(\tau) u(\tau) J_{T, i}^{(a, b)}(\tau) d \tau, \quad i=0,1, \ldots
$$

In practice, only the first $(n+1)$ terms of $J_{T, i}^{(a, b)}(\tau)$ are utilized. So, we have

$$
u(\tau) \simeq u_{n}(\tau)=\sum_{i=0}^{n} c_{i} J_{T, i}^{(a, b)}(\tau) \triangleq C^{T} \Psi_{T, n}(\tau)
$$


where

$$
C=\left[\begin{array}{llll}
c_{0} & c_{1} & \ldots & c_{n}
\end{array}\right]^{T},
$$

and

$$
\Psi_{T, n}(\tau)=\left[\begin{array}{llll}
J_{T, 0}^{(a, b)}(\tau) & J_{T, 1}^{(a, b)}(\tau) & \ldots & J_{T, n}^{(a, b)}(\tau)
\end{array}\right]^{T} .
$$

Similarly, any function $u(\xi, \tau)$ defined over $[0, L] \times[0, T]$ may be written via the double SJPs as follows:

$$
u(\xi, \tau) \simeq u_{m, n}(\xi, \tau)=\sum_{i=0}^{m} \sum_{j=0}^{n} u_{i j} J_{L, i}^{(a, b)}(\xi) J_{T, j}^{(a, b)}(\tau) \triangleq \Psi_{L, m}(\tau)^{T} \mathbf{U} \Psi_{T, n}(\tau),
$$

where $\mathbf{U}=\left[u_{i j}\right]$ is an $(m+1) \times(n+1)$ matrix with

$$
\begin{aligned}
u_{i j} & =\frac{1}{h_{L, i-1}^{(a, b)} h_{T, j-1}^{(a, b)}} \int_{0}^{L} \int_{0}^{T} w_{L}^{(a, b)}(\xi) w_{T}^{(a, b)}(\tau) u(\xi, \tau) J_{L, i-1}^{(a, b)}(\xi) J_{T, j-1}^{(a, b)}(\tau) d \tau d \xi \\
& i=1,2, \ldots, m+1, j=1,2, \ldots, n+1 .
\end{aligned}
$$

Remark 1 Note that the most commonly used SJPs are the shifted Legendre polynomials $P_{T, i}(\tau)$, the second, third and fourth kinds shifted Chebyshev polynomials $U_{T, i}(\tau), V_{T, i}(\tau)$ and $W_{T, i}(\tau)$. Meanwhile, we have

$$
\begin{aligned}
& P_{T, i}(\tau)=J_{T, i}^{(0,0)}(\tau), \quad U_{T, i}(\tau)=\frac{(i+1) ! \Gamma\left(\frac{1}{2}\right)}{\Gamma\left(i+\frac{3}{2}\right)} J_{T, i}^{\left(\frac{1}{2}, \frac{1}{2}\right)}(\tau), \\
& V_{T, i}(\tau)=\frac{(2 i) ! !}{(2 i-1) ! !} J_{T, i}^{\left(\frac{1}{2},-\frac{1}{2}\right)}(\tau), \quad W_{T, i}(\tau)=\frac{(2 i) ! !}{(2 i-1) ! !} J_{T, i}^{\left(-\frac{1}{2}, \frac{1}{2}\right)}(\tau) .
\end{aligned}
$$

\section{Operational matrices}

In the following, some novel matrix relations are obtained for the SJPs.

Definition 4.1 A set containing $(n+1)$ components of the monomials can be expressed by

$$
\Phi_{n}(\tau)=\left[\begin{array}{llll}
\varphi_{n, 0}(\tau) & \varphi_{n, 1}(\tau) & \ldots & \varphi_{n, n}(\tau)
\end{array}\right]^{T},
$$

where $\varphi_{n, i}(\tau)=\tau^{i}$ for $i=0,1, \ldots, n$.

Lemma 4.2 Let $\Psi_{T, n}(\tau)$ and $\Phi_{n}(\tau)$ be the basis vectors expressed in Eqs. (3.7) and (4.1), respectively. Then the following relation is valid between them:

$$
\Phi_{n}(\tau)=\mathbf{P}_{T, n} \Psi_{T, n}(\tau)
$$


where $\mathbf{P}_{T, n}=\left[p_{T, i j}\right]$ is an $(n+1)$ th-order square matrix with elements

$$
\begin{aligned}
& p_{T, i j}= \frac{T^{i-1}(2 j+a+b-1)(j-1) ! \Gamma(a+1)}{\Gamma(j+a)} \\
& \times \sum_{k=0}^{j-1}(-1)^{j+k-1} \frac{\Gamma(i+k+b) \Gamma(j+k+a+b)}{(j-k-1) ! k ! \Gamma(k+b+1) \Gamma(i+k+a+b+1)}, \\
& 1 \leq i, j \leq n+1 .
\end{aligned}
$$

Proof Any element $\varphi_{n, \hat{i}}(\tau), \hat{i}=0,1, \ldots, n$ of $\Phi_{n}(\tau)$ can be exactly expressed in terms of the SJPs as follows:

$$
\varphi_{n, \hat{i}}(\tau)=\sum_{\hat{j}=0}^{n} \hat{p}_{T, \hat{i}} J_{T, \hat{j}}^{(a, b)}(\tau) \triangleq \hat{P}_{T, \hat{i}}^{T} \Psi_{T, n}(\tau)
$$

where

$$
\hat{P}_{T, \hat{i}}^{T}=\left[\begin{array}{llll}
\hat{p}_{T, \hat{i} 0} & \hat{p}_{T, \hat{i} 1} & \cdots & \hat{p}_{T, \hat{i n}}
\end{array}\right],
$$

and according to Eq. (3.5), we have

$$
\hat{p}_{T, \hat{i} \hat{j}}=\frac{1}{h_{T, \hat{j}}^{(a, b)}} \int_{0}^{T} w_{T}^{(a, b)}(\tau) \varphi_{n, \hat{i}}(\tau) J_{T, \hat{j}}^{(a, b)}(\tau) d \tau, \quad 0 \leq \hat{j} \leq n .
$$

Regarding Eqs. (3.1), (3.3) and (4.1), and the definition of $w_{T}^{(a, b)}(\tau)$, one has

$$
\begin{aligned}
\frac{1}{h_{T, \hat{j}}^{(a, b)}} \int_{0}^{T} w_{T}^{(a, b)}(\tau) \varphi_{h, \hat{i}}(\tau) J_{T, \hat{j}}^{(a, b)}(\tau) d \tau \\
=\frac{T^{\hat{i}}(2 \hat{j}+a+b+1) \hat{j} ! \Gamma(a+1)}{\Gamma(\hat{j}+a+1)} \\
\quad \times \sum_{k=0}^{\hat{j}}(-1)^{\hat{j}+k} \frac{\Gamma(\hat{i}+k+b+1) \Gamma(\hat{j}+k+a+b+1)}{(\hat{j}-k) ! k ! \Gamma(k+b+1) \Gamma(\hat{i}+k+a+b+2)} .
\end{aligned}
$$

Thus, from Eqs. (4.5) and (4.6), we obtain

$$
\begin{aligned}
\hat{p}_{T, \hat{j}} & =\frac{T^{\hat{i}}(2 \hat{j}+a+b+1) \hat{j} ! \Gamma(a+1)}{\Gamma(\hat{j}+a+1)} \sum_{k=0}^{\hat{j}}(-1)^{\hat{j}+k} \frac{\Gamma(\hat{i}+k+b+1) \Gamma(\hat{j}+k+a+b+1)}{(\hat{j}-k) ! k ! \Gamma(k+b+1) \Gamma(\hat{i}+k+a+b+2)} \\
& \leq \hat{j} \leq n
\end{aligned}
$$

Eventually, by change of indices $i=\hat{i}+1$ and $j=\hat{j}+1$ together with replacing $\hat{P}_{T, i-1}^{T}$ and $\hat{p}_{T,(i-1)(j-1)}$, respectively, by $\mathbf{P}_{T, i}^{T}$ and $p_{T, i j}$ the proof is completed. 
As an illustrative example, for $(a=1, b=2), T=2$ and $n=5$, we have

$$
\mathbf{P}_{2,5}=\left(\begin{array}{cccccc}
1 & 0 & 0 & 0 & 0 & 0 \\
\frac{6}{5} & \frac{2}{5} & 0 & 0 & 0 & 0 \\
\frac{8}{5} & \frac{32}{35} & \frac{4}{21} & 0 & 0 & 0 \\
\frac{16}{7} & \frac{12}{7} & \frac{40}{63} & \frac{2}{21} & 0 & 0 \\
\frac{24}{7} & \frac{64}{21} & \frac{32}{21} & \frac{32}{77} & \frac{8}{165} & 0 \\
\frac{16}{3} & \frac{16}{3} & \frac{320}{99} & \frac{40}{33} & \frac{112}{429} & \frac{32}{1287}
\end{array}\right)
$$

Lemma 4.3 Assume that $\Phi_{n}(\tau)$ is the vector defined in Eq. (4.1). Then we have

$$
\frac{d \Phi_{n}(\tau)}{d \tau}=\mathbb{D}_{n}^{(1)} \Phi_{n}(\tau)
$$

where $\mathbb{D}^{(1)}$ is a strictly lower triangular matrix of order $(n+1)$ with

$$
\left[\mathbb{D}_{n}^{(1)}\right]_{i j}= \begin{cases}0, & i=1,1 \leq j \leq n+1 \\ i-1, & 2 \leq i \leq n+1,1 \leq j \leq n+1, i-j=1\end{cases}
$$

Generally, we have

$$
\frac{d^{r} \Psi_{n}(\tau)}{d \tau^{r}}=\mathbb{D}_{n}^{(r)} \Psi_{n}(\tau)
$$

where $\mathbb{D}_{n}^{(r)}$ is the rth power of $\mathbb{D}_{n}^{(1)}$.

Proof The proof is clear by considering the definition of these polynomials.

Lemma 4.4 If $\Phi_{n}(\tau)$ is the basis vector given in Eq. (4.1) and $\omega:[0, L] \times[0, T] \rightarrow(0,1)$ is continuous, then

$$
\frac{\partial^{\omega(\xi, \tau)} \Phi_{n}(\tau)}{\partial \tau^{\omega(\xi, \tau)}}=\mathbb{Z}_{n}^{(\omega(\xi, \tau))} \Phi_{n}(\tau)
$$

where $\mathbb{Z}_{n}^{(\omega(\xi, \tau))}$ is a strictly lower triangular matrix of $(n+1)$ with

$$
\left[\mathbb{Z}_{n}^{(\omega(\xi, \tau))}\right]_{i j}= \begin{cases}0, & i=1,1 \leq j \leq n+1 \\ \frac{(i-1) ! \mathbf{C}(\omega(\xi, \tau))}{1-\omega(\xi, \tau)} \mathbf{E}_{\omega(\xi, \tau), i}\left(\frac{-\omega(\xi, \tau) \tau(\xi, \tau)}{1-\omega(\xi, \tau)}\right), & 2 \leq i \leq n+1,1 \leq j \leq n+1, i=j\end{cases}
$$

Proof Regarding Corollary 2.4, the proof is clear.

Theorem 4.5 If $\Psi_{T, n}(\tau)$ is the SJPs vector introduced in Eq. (3.7), then

$$
\frac{d \Psi_{T, n}(\tau)}{d \tau}=\mathbf{D}_{T, n}^{(1)} \Psi_{T, n}(\tau)
$$

where $\mathbf{D}_{T, n}^{(1)}$ is a matrix (the derivative operational matrix of the SJPs) of order $(n+1)$ as follows:

$$
\mathbf{D}_{T, n}^{(1)}=\mathbf{P}_{T, n}^{-1} \mathbb{D}_{n}^{(1)} \mathbf{P}_{T, n}
$$


Generally, we have

$$
\frac{d^{r} \Psi_{T, n}(\tau)}{d \tau^{r}}=\mathbf{D}_{T, n}^{(r)} \Psi_{T, n}(\tau)
$$

where $\mathbf{D}_{T, n}^{(r)}$ is the rth power of $\mathbf{D}_{T, n}^{(1)}$.

Theorem 4.6 If $\Psi_{T, n}(\tau)$ is the SJPs vector given in Eq. (3.7) and $\omega:[0, L] \times[0, T] \rightarrow(0,1)$ is a continuous function, then

$$
\frac{\partial^{\omega(\xi, \tau)} \Psi_{T, n}(\tau)}{\partial \tau^{\omega(\xi, \tau)}}=\mathbf{Z}_{T, n}^{(\omega(\xi, \tau))} \Psi_{T, n}(\tau)
$$

where $\mathbf{Z}_{T, n}^{(\omega(\xi, \tau))}$ is a matrix (named the VO fractional derivative operator matrix of order $\omega(\xi, \tau)$ of the SJPS) of order $(n+1)$ as follows:

$$
\mathbf{Z}_{T, n}^{(\omega(\xi, \tau))}=\mathbf{P}_{T, n}^{-1} \mathbb{Z}_{n}^{(\omega(\xi, \tau))} \mathbf{P}_{T, n}
$$

Proof Regarding Lemmas 4.2 and 4.4, the proof is straightforward.

\section{The proposed method}

For solving the VO fractional model given in Eq. (1.1), by approximating the solution in terms of the SJPs, we have

$$
u(\xi, \tau) \simeq u_{m, n}(\xi, \tau)=\sum_{i=0}^{m} \sum_{j=0}^{n} u_{i j} j_{L, i}^{(a, b)}(\xi) J_{T, j}^{(a, b)}(\tau) \triangleq \Psi_{L, m}(\xi)^{T} \mathbf{U} \Psi_{T, n}(\tau)
$$

where $\mathbf{U}=\left[u_{i j}\right]$ is an unknown matrix with $(m+1) \times(n+1)$ elements, and $\Psi_{L, m}(\xi)$ and $\Psi_{T, n}(\tau)$ are the vectors expressed in Eq. (3.8). Regarding Theorem 4.5, we obtain

$$
\frac{\partial u(\xi, \tau)}{\partial \xi} \simeq \Psi_{L, m}(\xi)^{T}\left(\mathbf{D}_{L, m}^{(1)}\right)^{T} \mathbf{U} \Psi_{T, n}(\tau)
$$

and

$$
\frac{\partial^{2} u(\xi, \tau)}{\partial \xi^{2}} \simeq \Psi_{L, m}(\xi)^{T}\left(\mathbf{D}_{L, m}^{(2)}\right)^{T} \mathbf{U} \Psi_{T, n}(\tau)
$$

Also, from Theorem 4.6, we have

$$
\frac{\partial^{\omega(\xi, \tau)} u(\xi, \tau)}{\partial \tau^{\omega(\xi, \tau)}} \simeq \Psi_{L, m}(\xi)^{T} \mathbf{U} \mathbf{Z}_{T, n}^{(\omega(\xi, \tau))} \Psi_{T, n}(\tau)
$$

By inserting Eqs. (5.1)-(5.4) into Eq. (1.1), we introduce the residual function

$$
\begin{aligned}
\mathbf{R}(\xi, \tau) \triangleq & \Psi_{L, m}(\xi)^{T}\left[\mathbf{U} \mathbf{Z}_{T, n}^{(\omega(\xi, \tau))}-\left(\mathbf{D}_{L, m}^{(2)}\right) \mathbf{U}-\frac{\lambda}{\xi}\left(\mathbf{D}_{L, m}^{(1)}\right) \mathbf{U}\right] \Psi_{T, n}(\tau) \\
& -\mathcal{G}\left(\xi, \tau, \Psi_{L, m}(\xi)^{T} \mathbf{U} \Psi_{T, n}(\tau)\right)-f(\xi, \tau) .
\end{aligned}
$$


On the other hand, from Eqs. (1.2), (1.3) and (5.1), we have

$$
\Psi_{L, m}(\xi)^{T} \mathbf{U} \Psi_{T, n}(0)-g(\xi) \triangleq \Lambda_{1}(\xi) \simeq 0
$$

and

$$
\begin{aligned}
& \Psi_{L, m}(0)^{T} \mathbf{U} \Psi_{T, n}(\tau)-h_{0}(\tau) \triangleq \Lambda_{2}(\tau) \simeq 0, \\
& \Psi_{L, m}(L)^{T} \mathbf{U} \Psi_{T, n}(\tau)-h_{1}(\tau) \triangleq \Lambda_{3}(\tau) \simeq 0 .
\end{aligned}
$$

Eventually, the following system with $(m+1) \times(n+1)$ equations can be generated from Eqs. (5.5)-(5.7):

$$
\begin{cases}\mathbf{R}\left(\xi_{i}, \tau_{j}\right)=0, & 2 \leq i \leq m, 2 \leq j \leq n+1 \\ \Lambda_{1}\left(\xi_{i}\right)=0, & 1 \leq i \leq m+1 \\ \Lambda_{2}\left(\tau_{j}\right)=0, & 2 \leq j \leq n+1 \\ \Lambda_{3}\left(\tau_{j}\right)=0, & 2 \leq j \leq n+1,\end{cases}
$$

where $\xi_{i}=\frac{L}{2}\left(1-\cos \left(\frac{(2 i-1) \pi}{2(m+1)}\right)\right)$ for $i=1,2, \ldots, m+1$ and $\tau_{j}=\frac{T}{2}\left(1-\cos \left(\frac{(2 j-1) \pi}{2(n+1)}\right)\right)$ for $j=$ $1,2, \ldots, n+1$. The above system should be solved for computing the matrix $\mathbf{U}$ in Eq. (5.1), and consequently obtaining an approximate solution for the problem.

\section{Test problems}

Here, the accuracy of the explained algorithm is studied by solving some numerical test problems. Maple 18 (with 25 decimal digits) is used for numerical simulations. The experimental convergence order (ECO) of the expressed scheme is obtained by

$$
\mathrm{ECO}=\log _{\frac{\mathcal{S}_{1}}{\mathcal{S}_{2}}}\left(\frac{\varepsilon_{2}}{\varepsilon_{1}}\right)
$$

where $\varepsilon_{1}$ and $\varepsilon_{2}$ express the maximum absolute error (MAE) arisen in the first and second implementations, respectively. Moreover, $\mathcal{S}_{i}=\left(m_{i}+1\right)\left(n_{i}+1\right)$ for $i=1,2$ denote the number of the GJPs applied in the first and second experiments, respectively. Meanwhile, the series in the Mittag-Leffler function is truncated after the 35th term.

Example 1 First, consider the equation

$$
\frac{\partial^{\omega(\xi, \tau)} u(\xi, \tau)}{\partial \tau^{\omega(\xi, \tau)}}=\frac{\partial^{2} u(\xi, \tau)}{\partial \xi^{2}}+\frac{1}{\xi} \frac{\partial u(\xi, \tau)}{\partial \xi}+e^{u(\xi, \tau)}-e^{\frac{1}{2} u(\xi, \tau)}+f(\xi, \tau), \quad(\xi, \tau) \in[0,1] \times[0,1],
$$

where

$$
f(\xi, \tau)=\frac{1}{\left(\tau \xi^{2}+5\right)^{\frac{1}{2}}}-\frac{\xi^{2}-4 \tau+1}{\tau \xi^{2}+5}-\frac{4 \xi^{2} \tau^{2}}{\left(\tau \xi^{2}+5\right)^{2}},
$$

with the IBCs

$$
\begin{aligned}
& u(\xi, 0)=-\ln (5), \\
& u(0, \tau)=-\ln (5), \quad u(1, \tau)=-\ln (5+\tau) .
\end{aligned}
$$



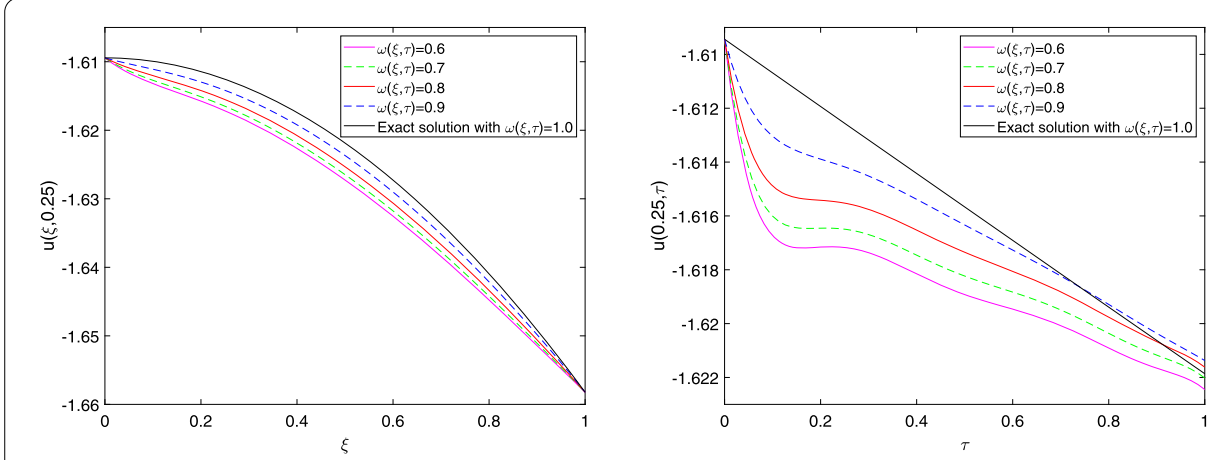

Figure 1 Behavior of the numerical solutions $u(\zeta, 0.25)$ (left) and $u(0.25, \tau)$ (right) for some functions $\omega(\zeta, \tau)$ in Example 1
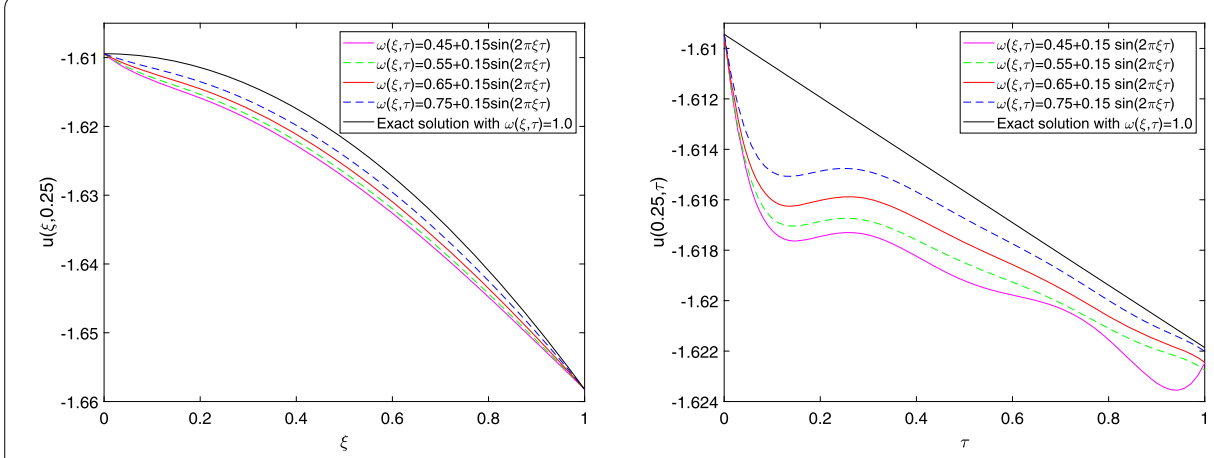

Figure 2 Behavior of the numerical solutions $u(\zeta, 0.25)$ (left) and $u(0.25, \tau)$ (right) for some functions $\omega(\zeta, \tau)$ in Example 1

The exact solution of this example when $\omega(\xi, \tau)=1$ is $u(\xi, \tau)=-\ln \left(5+\xi^{2} \tau\right)$ [34]. The approach expressed in the previous section with $(a=b=0)$ and $(m=n=7)$ is applied for this example. The numerical treatments of the results for $u(\xi, 0.25)$ and $u(0.25, \tau)$ with some selections $\omega(\xi, \tau)$ are shown in Figs. 1 and 2.

Example 2 Consider the equation

$$
\begin{aligned}
\frac{\partial^{\omega(\xi, \tau)} u(\xi, \tau)}{\partial \tau^{\omega(\xi, \tau)}=} & \frac{\partial^{2} u(\xi, \tau)}{\partial \xi^{2}}+\frac{\lambda}{\xi} \frac{\partial u(\xi, \tau)}{\partial \xi}-\theta \tau(\xi \tau)^{\theta-2}\left(\xi^{2}-\tau(\lambda+\theta-1)\right) e^{u(\xi, \tau)} \\
& -\theta^{2} \tau^{2}(\xi \tau)^{2 \theta-2} e^{2 u(\xi, \tau)}
\end{aligned}
$$

where $(\xi, \tau) \in[0,1] \times[0,1], \lambda$ and $\theta$ are constants, subject to the IBCs

$$
\begin{aligned}
& u(\xi, 0)=-\ln (3), \\
& u(0, \tau)=-\ln (3), \quad u(1, \tau)=-\ln \left(3+\tau^{\theta}\right) .
\end{aligned}
$$

The exact solution of this example when $\omega(\xi, \tau)=1$ is $u(\xi, \tau)=-\ln \left(3+(\xi \tau)^{2}\right)$ [35]. The method introduced in Sect. 5 with $\left(a=1, b=\frac{1}{2}\right)$ and $(m=8, n=7)$ is utilized for this example. The behavior of the numerical results for two cases $(\lambda=1, \theta=1)$ and $(\lambda=2, \theta=2)$ are illustrated in Figs. 3 and 4. 

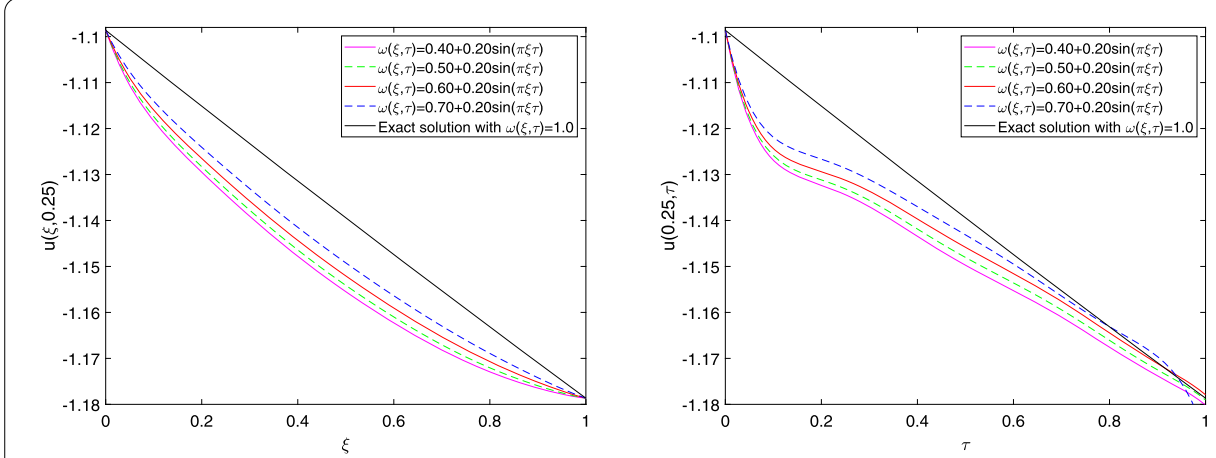

Figure 3 Behavior of the numerical solutions $u(\zeta, 0.25)$ (left) and $u(0.25, \tau)$ (right) where $(\lambda=1, \theta=1)$ for some functions $\omega(\zeta, \tau)$ in Example 2
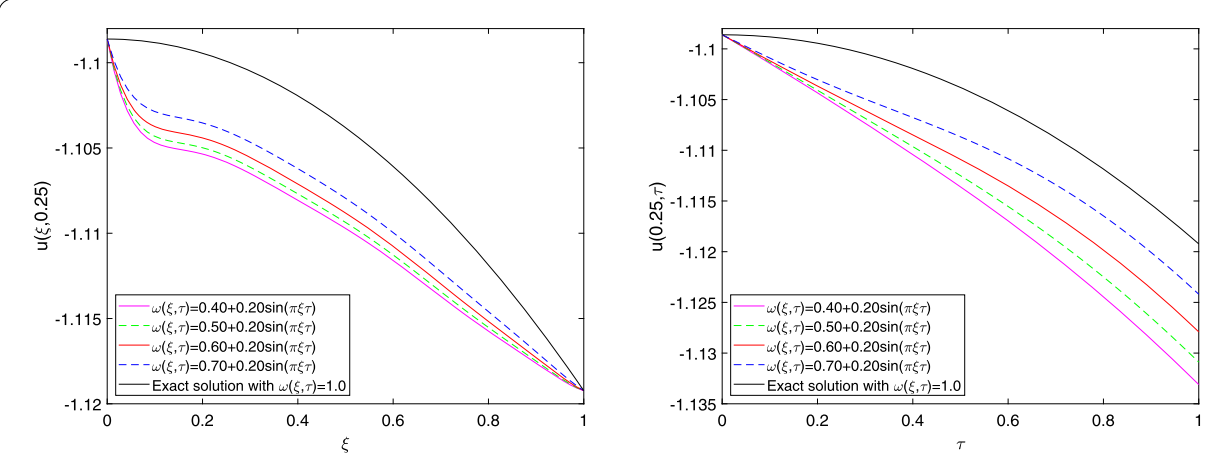

Figure 4 Behavior of the numerical solutions $u(\zeta, 0.25)$ (left) and $u(0.25, \tau)$ (right) where $(\lambda=2, \theta=2)$ for some functions $\omega(\zeta, \tau)$ in Example 2

Example 3 Consider the equation

$$
\begin{aligned}
& \frac{\partial^{\omega(\xi, \tau)} u(\xi, \tau)}{\partial \tau^{\omega(\xi, \tau)}}=\frac{\partial^{2} u(\xi, \tau)}{\partial \xi^{2}}+\frac{3}{\xi} \frac{\partial u(\xi, \tau)}{\partial \xi}+\left(1+e^{-\xi \tau}\right) \sin (u(\xi, \tau))+f(\xi, \tau), \\
& (\xi, \tau) \in[0,2] \times[0,1],
\end{aligned}
$$

where

$$
\begin{aligned}
f(\xi, \tau)= & -\frac{\mathbf{C}(\omega(\xi, \tau)) \tau \sin (\xi)}{1-\omega(\xi, \tau)} \sum_{k=0}^{\infty}(-\tau)^{k} \mathbf{E}_{\omega(\xi, \tau), k+2}\left(\frac{-\omega(\xi, \tau) \tau^{\omega(\xi, \tau)}}{1-\omega(\xi, \tau)}\right) \\
& +\left(\sin (\xi)-\frac{3}{\xi} \cos (\xi)\right) e^{-\tau}-\left(1+e^{-\xi \tau}\right) \sin \left(\sin (\xi) e^{-\tau}\right),
\end{aligned}
$$

with the IBCs

$$
\begin{aligned}
& u(\xi, 0)=\sin (\xi), \\
& u(0, \tau)=0, \quad u(2, \tau)=\sin (2) e^{-\tau} .
\end{aligned}
$$

The analytic solution of this equation is $u(\xi, \tau)=\sin (\xi) e^{-\tau}$. The expressed algorithm for some values $(a, b)$ is utilized for this equation where $\omega(\xi, \tau)=0.65+0.25 \sin (2 \xi \tau)$. The 
Table 1 Numerical results obtained for Example 3 with some values $(a, b)$

\begin{tabular}{|c|c|c|c|c|c|c|c|c|c|}
\hline \multirow[t]{2}{*}{$n$} & \multirow[t]{2}{*}{$n$} & \multicolumn{2}{|l|}{$(a=0, b=0)$} & \multicolumn{2}{|l|}{$\left(a=\frac{1}{2}, b=\frac{1}{2}\right)$} & \multicolumn{2}{|c|}{$\left(a=\frac{1}{2}, b=-\frac{1}{2}\right)$} & \multicolumn{2}{|c|}{$\left(a=-\frac{1}{2}, b=\frac{1}{2}\right)$} \\
\hline & & $\overline{M A E}$ & ECO & $\overline{M A E}$ & ECO & $\overline{M A E}$ & ECO & MAE & ECO \\
\hline 5 & 5 & 1.157 & - & $1.1577 \mathrm{E}$ & - & 1.1577 & - & 1.1577E-04 & - \\
\hline 7 & 7 & 5.3 & 09.3 & 5. & 09. & 7 & & 4E-07 & 09. \\
\hline 9 & 9 & 1. & 13. & 1. & 13 & 99 & 13 & $6 \mathrm{E}-09$ & 142 \\
\hline 11 & 11 & 1.3455E-12 & 18.9318 & $1.3455 E-12$ & 18.9318 & $1.3455 E-12$ & 18.9318 & 1.3455E-12 & 18.9318 \\
\hline
\end{tabular}

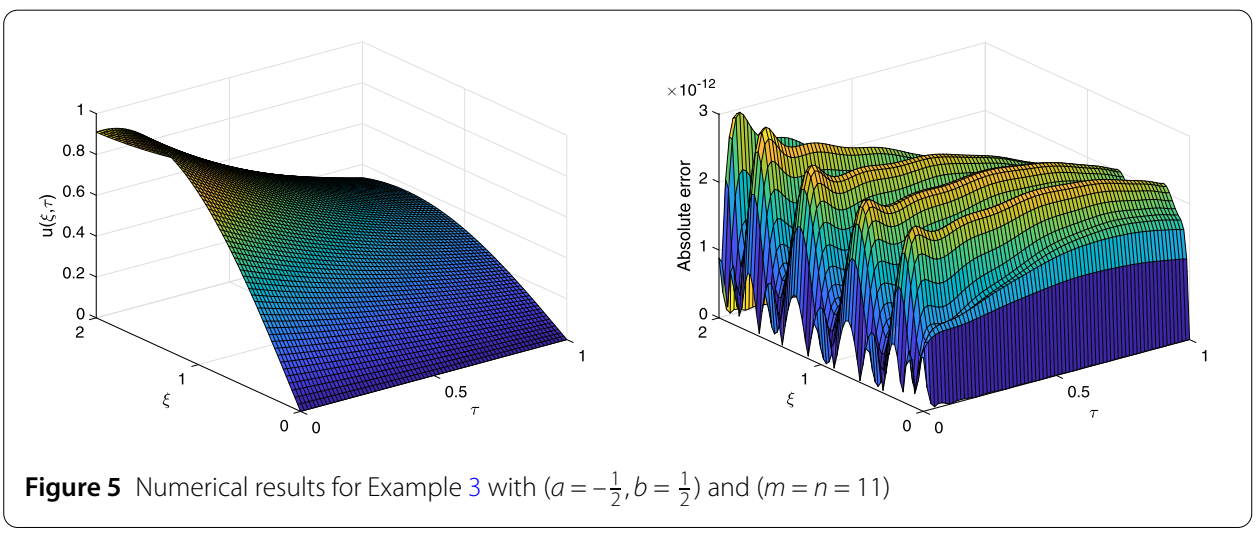

obtained results are shown in Table 1 . The obtained results for the case of $\left(a=-\frac{1}{2}, b=\frac{1}{2}\right)$ and $(m=n=11)$ are illustrated in Fig. 5 . From Table 1, it can be seen that the numerical results are the same for all selections $(a, b)$. Figure 5 shows the high precision of the presented scheme. Note that the infinite series in the right side is truncated after the 25th term. This assumption is also used for the next example.

Example 4 Consider the equation

$$
\begin{aligned}
& \frac{\partial^{\omega(\xi, \tau)} u(\xi, \tau)}{\partial \tau^{\omega(\xi, \tau)}}=\frac{\partial^{2} u(\xi, \tau)}{\partial \xi^{2}}+\frac{2}{\xi} \frac{\partial u(\xi, \tau)}{\partial \xi}+\sin (\xi \tau) e^{u(\xi, \tau)}+e^{\xi \tau-u(\xi, \tau)}+f(\xi, \tau), \\
& (\xi, \tau) \in\left[0, \frac{3}{2}\right] \times\left[0, \frac{3}{4}\right]
\end{aligned}
$$

where

$$
\begin{aligned}
f(\xi, \tau)= & \frac{\mathbf{C}(\omega(\xi, \tau)) \tau \cos (\xi)}{1-\omega(\xi, \tau)} \sum_{k=0}^{\infty}\left(-\tau^{2}\right)^{k} \mathbf{E}_{\omega(\xi, \tau), 2 k+2}\left(\frac{-\omega(\xi, \tau) \tau^{\omega(\xi, \tau)}}{1-\omega(\xi, \tau)}\right) \\
& +\left(\cos (\xi)+\frac{2}{\xi} \sin (\xi)\right) \sin (\tau)-\sin (\xi \tau) e^{\cos (\xi) \sin (\tau)}-e^{\xi \tau-\cos (\xi) \sin (\tau)}
\end{aligned}
$$

The IBCs can be identified from the analytic solution

$$
u(\xi, \tau)=\cos (\xi) \sin (\tau) .
$$

We have used the introduced method with $(a=2, b=1)$ for solving this example with some functions $\omega(\xi, \tau)$. The obtained results are reported in Table 2. Plots of the obtained results where $(m=12, n=11)$ and $\omega(\xi, \tau)=0.75-0.25 e^{-\xi \tau}$ are shown in Fig. 6 . The obtained results show the high-order convergence of the presented algorithm for all studied cases. 
Table 2 Numerical results obtained for Example 4 with some selections $\omega(\xi, \tau)$

\begin{tabular}{|c|c|c|c|c|c|c|c|c|c|}
\hline \multirow[t]{2}{*}{$m$} & \multirow[t]{2}{*}{$n$} & \multicolumn{2}{|c|}{$\begin{array}{l}\omega(\xi, \tau)= \\
0.75-0.25 e^{-\xi \tau}\end{array}$} & \multicolumn{2}{|c|}{$\begin{array}{l}\omega(\xi, \tau)= \\
0.55+0.35 \cos (\pi \xi \tau)\end{array}$} & \multicolumn{2}{|c|}{$\begin{array}{l}\omega(\xi, \tau)= \\
0.65+0.25 \sin (\pi \xi \tau)\end{array}$} & \multicolumn{2}{|c|}{$\begin{array}{l}\omega(\xi, \tau)= \\
(1+0.75 \sin (3 \pi \xi \tau)) / 2\end{array}$} \\
\hline & & MAE & ECO & MAE & ECO & MAE & ECO & MAE & ECO \\
\hline 5 & 5 & 1.0047 & - & 1.0144 & - & $9.6282 \mathrm{E}$ & - & $1.0108 \mathrm{E}-05$ & - \\
\hline 7 & 7 & $2.6408 \mathrm{E}$ & 10.3 & $2.6744 \mathrm{E}$ & 10. & 4.3907 & 17.3 & 2.665 & 10. \\
\hline 9 & 9 & $3.9134 \mathrm{E}-11$ & 14.5969 & $3.9592 \mathrm{E}-11$ & 14.5992 & 3.7373E & 05.5204 & $4.0509 \mathrm{E}-11$ & 14.5403 \\
\hline 11 & 11 & $2.6345 \mathrm{E}-15$ & 26.3437 & $1.3736 \mathrm{E}-15$ & 28.1616 & $3.0320 \mathrm{E}-16$ & 32.1466 & $2.2488 \mathrm{E}-15$ & 26.8725 \\
\hline
\end{tabular}

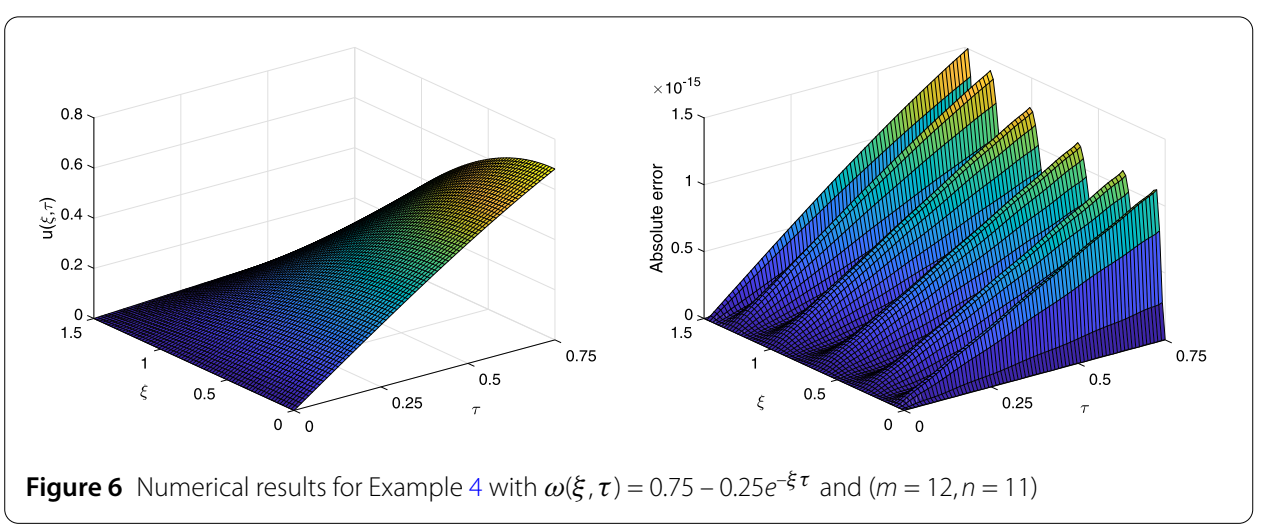

\section{Conclusion}

In this paper, a novel version of nonlinear singular Emden-Fowler equation generated by employing the concept of fractional derivative of variable order with non-singular kernel function. A computational method using the shifted Jacobi polynomials truncated series developed for this category of problems. In the established approach, by using the operational matrices of derivatives (ordinary and of variable order) of the shifted Jacobi polynomials, the problem converts into a system of algebraic equations. Several test examples investigated to examine the accuracy of the present approach. The obtained results manifest that the proposed approach is very accurate for such problems.

\section{Acknowledgements}

We thank the reviewers for their valuable comments and suggestions. In addition, we would like to thank professor F.M. Maalek Ghaini (Yazd University, Iran) for his careful review of our work.

Funding

The financial assistance is not applicable.

Availability of data and materials

Data sharing is not applicable to this study.

\section{Competing interests}

The authors declare that they have no competing interests.

Authors' contributions

All authors contributed equally to the writing of this paper. All authors read and approved the final manuscript.

\section{Author details}

'Department of Mathematics, Shiraz University of Technology, Shiraz, Iran. ²Department of Applied Mathematics, Xi'an Jiaotong-Liverpool University, Suzhou 215123, Jiangsu, China. ${ }^{3}$ Faculty of Natural and Agricultural Sciences, University of the Free State, Bloemfontein, South Africa. ${ }^{4}$ Department of Medical Research, China Medical University Hospital, China Medical University, Taichung, Taiwan.

\section{Publisher's Note}

Springer Nature remains neutral with regard to jurisdictional claims in published maps and institutional affiliations. 
Received: 4 June 2020 Accepted: 17 March 2021 Published online: 25 March 2021

\section{References}

1. Podlubny, I.: Fractional Differential Equations. Academic Press, San Diego (1999)

2. Baleanu, D., Jajarmi, A., Sadat Sajjadi, S., Asad, J.: The fractional features of a harmonic oscillator with position-dependent mass. Commun. Theor. Phys. 75(5), 055002 (2020)

3. Sadat Sajjadi, S., Baleanu, D., Jajarmi, A., Mohammadi Pirouz, H.: A new adaptive synchronization and hyperchaos control of a biological snap oscillator. Chaos Solitons Fractals 138, 109919 (2020)

4. Jajarmi, A., Yusuf, A., Baleanu, D., Inc, M.: A new fractional HRSV model and its optimal control: a non-singular operator approach. Phys. A, Stat. Mech. Appl. 547, 123860 (2020)

5. Yıldız, T.A., Jajarmi, A., Yıldız, B., Baleanu, D.: New aspects of time fractional optimal control problems within operators with nonsingular kernel. Discrete Contin. Dyn. Syst., Ser. S 13(3), 407-428 (2020)

6. Baleanu, D., Jajarmi, A., Mohammadi, H., Rezapour, S.: A new study on the mathematical modelling of human liver with Caputo-Fabrizio fractional derivative. Chaos Solitons Fractals 134, 109705 (2020)

7. Jajarmi, A., Baleanu, D., Sadat Sajjadi, S., Asad, J.H.: A new feature of the fractional Euler-Lagrange equations for a coupled oscillator using a nonsingular operator approach. Front. Phys. 7, 196 (2019)

8. Atangana, A., Baleanu, D.: New fractional derivatives with non-local and non-singular kernel: theory and application to heat transfer model. Therm. Sci. 20(2), 763-769 (2016)

9. Atangana, A.: Non validity of index law in fractional calculus: a fractional differential operator with Markovian and non-Markovian properties. Physica A 505, 688-706 (2018)

10. Caputo, M., Fabrizio, M.: A new definition of fractional derivative without singular kernel. Prog. Fract. Differ. Appl. 1(2), 73-85 (2015)

11. Heydari, M.H., Hosseininia, M.H.: A new variable-order fractional derivative with non-singular Mittag-Leffler kernel: application to variable-order fractional version of the 2D Richard equation. Eng. Comput. (2020). https://doi.org/10.1007/s00366-020-01121-9

12. Bahaa, G.M.: Optimal control problem for variable-order fractional differential systems with time delay involving Atangana-Baleanu derivatives. Chaos Solitons Fractals 122, 129-142 (2019)

13. Sun, H.G., Chen, W., Wei, H., Chen, Y.Q.: A comparative study of constant-order and variable-order fractional models in characterizing memory property of systems. Eur. Phys. J. Spec. Top. 193, 185-192 (2011)

14. Roohi, R., Heydari, M.H., Sun, H.G.: Numerical study of unsteady natural convection of variable-order fractional Jeffrey nanofluid over an oscillating plate in a porous medium involved with magnetic, chemical and heat absorption effects using Chebyshev cardinal functions. Eur. Phys. J. Plus 134, 535 (2019)

15. Roohi, R., Heydari, M.H., Bavi, O., Emdad, H.: Chebyshev polynomials for generalized Couette flow of fractional Jeffrey nanofluid subjected to several thermochemical effects. Eng. Comput. (2019). https://doi.org/10.1007/s00366-019-00843-9

16. Hosseininia, M., Heydari, M.H., Rouzegar, J., Cattani, C.: A meshless method to solve nonlinear variable-order time fractional 2D reaction-diffusion equation involving Mittag-Leffler kernel. Eng. Comput. (2019) https://doi.org/10.1007/s00366-019-00852-8

17. Zúniga-Aguilar, C.J., Romero-Ugalde, H.M., Gómez-Aguilar, J.F., Escobar-Jiménez, R.F., Valtierra-Rodríguez, M.: Solving fractional differential equations of variable-order involving operators with Mittag-Leffler kernel using artificial neural networks. Chaos Solitons Fractals 103, 382-403 (2018)

18. Coronel-Escamilla, A., Gómez-Aguilar, J.F., Torres, L., Escobar-Jiménez, R.F.: A numerical solution for a variable-order reaction-diffusion model by using fractional derivatives with non-local and non-singular kernel. Phys. A, Stat. Mech. Appl. 491, 406-424 (2018)

19. Gómez-Aguilar, J.F., Atangana, A.: Time-fractional variable-order telegraph equation involving operators with Mittag-Leffler kernel. J. Electromagn. Waves Appl. 33(2), 165-175 (2019)

20. Solís-Pérez, J.E., Gómez-Aguilar, J.F., Atangana, A.: Novel numerical method for solving variable-order fractional differential equations with power, exponential and Mittag-Leffler laws. Chaos Solitons Fractals 114, 175-185 (2018)

21. Hossininia, M., Heydari, M.H.: Meshfree moving least squares method for nonlinear variable-order time fractional 2D telegraph equation involving Mittag-Leffler non-singular kernel. Chaos Solitons Fractals 127, $389-399$ (2019)

22. Hossininia, M., Heydari, M.H.: Legendre wavelets for the numerical solution of nonlinear variable-order time fractional $2 \mathrm{D}$ reaction-diffusion equation involving Mittag-Leffler non-singular kernel. Chaos Solitons Fractals 127, 400-407 (2019)

23. Heydari, M.H.: Chebyshev cardinal functions for a new class of nonlinear optimal control problems generated by Aatangana-Baleanu-Caputo variable-order fractional derivative. Chaos Solitons Fractals 130, 109401 (2020)

24. Heydari, M.H.: A cardinal approach for nonlinear variable-order time fractional Schrödinger equation defined by Atangana-Baleanu-Caputo derivative. Chaos Solitons Fractals 128, 339-348 (2019)

25. Doha, E.H., Bahrawy, A.H., Hafez, R.M.: A Jacobi dual-Petrov-Galerkin method for third- and fifth-order differential equations. Math. Comput. Model. 53, 1820-1832 (2011)

26. Canuto, C., Hussaini, M., Quarteroni, A., Zang, T.: Spectral Methods in Fluid Dynamics. Springer, Berlin (1988)

27. Wu, Q., Zeng, X.: Jacobi collocation methods for solving generalized space-fractional Burgers' equations. Commun. Appl. Math. Comput. (2019). https://doi.org/10.1007/s42967-019-00053-6

28. Jaiswal, S., Das, S.: Numerical solution of linear/nonlinear fractional order differential equations using Jacobi operational matrix. Int. J. Appl. Comput. Math. 5, 42 (2019)

29. Abdelkawy, M.A., Lopes, A.M., Zaky, M.A.: Shifted fractional Jacobi spectral algorithm for solving distributed order time-fractional reaction-diffusion equations. Discrete Appl. Math. 38, 81 (2019)

30. Zakya, M.A., Doha, E.H., Tenreiro Machado, J.A.: A spectral framework for fractional variational problems based on fractional Jacobi functions. Appl. Numer. Math. 132, 51-72 (2018)

31. Chandrasekhar, S.: Introduction to the Study of Stellar Structure. Dover, New York (1967)

32. Davis, H.T.: Introduction to Nonlinear Differential and Integral Equations. Dover, New York (1962)

33. Richardson, O.U.: The Emission of Electricity from Hot Bodies. Longmans, Green, London (1921) 
34. Mohammadi, A., Aghazadeh, N., Rezapour, Sh.: Haar wavelet collocation method for solving singular and nonlinear fractional time-dependent Emden-Fowler equations with initial and boundary conditions. Math. Sci. 13, 255-265 (2019)

35. Singh, R., Singh, S., Wazwaz, A.M.: A modified homotopy perturbation method for singular time dependent Emden-Fowler equations with boundary conditions. J. Math. Chem. 54, 918-931 (2016)

36. Chowdhury, M.S.H., Hashim, I.: Solutions of time-dependent Emden-Fowler type equations by homotopy-perturbation method. Phys. Lett. A 13, 305-313 (2007)

37. Zúñiga Aguilar, C.J., Coronel-Escamilla, A., Gómez Aguilar, J.F., Alvarado-Martínez, V.M., Romero-Ugalde, H.M.: New numerical approximation for solving fractional delay differential equations of variable order using artificial neural networks. Eur. Phys. J. Plus 133, 75 (2018)

38. Bhrawy, A.H., Zaky, M.A.: A method based on the Jacobi tau approximation for solving multi-term time-space fractional partial differential equations. J. Comput. Phys. 281, 876-895 (2015)

\section{Submit your manuscript to a SpringerOpen ${ }^{\circ}$ journal and benefit from:}

- Convenient online submission

- Rigorous peer review

- Open access: articles freely available online

- High visibility within the field

- Retaining the copyright to your article

Submit your next manuscript at $\gg$ springeropen.com 